\title{
40 Jahre Aktuelle Dermatologie
}

\author{
40 Years of Aktuelle Dermatologie
}

Autoren

Institute
C. Bayerl' ${ }^{1}$, E. G. Jung ${ }^{2}$

Klinik für Dermatologie und Allergologie, HSK, Dr.-Horst-Schmidt-Kliniken GmbH, Wiesbaden

2 Prof. em. der Universitätshautklinik Mannheim, Fakultät für Klinische Medizin der Ruprecht-Karls-Universität Heidelberg
Bibliografie

DOI http://dx.doi.org/

$10.1055 / \mathrm{s}-0034-1377312$

Akt Dermatol 2014; 40: 269-272

(c) Georg Thieme Verlag KG

Stuttgart · New York

ISSN 0340-2541

Korrespondenzadresse

Prof. Dr. med.

Christiane Bayerl

Klinik für Dermatologie

und Allergologie, HSK

Dr.-Horst-Schmidt-Kliniken GmbH

Ludwig-Erhard-Straße 100

65199 Wiesbaden

Christiane.BayerI@HSK-

Wiesbaden.de

\section{Zusammenfassung \\ $\nabla$}

Die Zeitschrift Aktuelle Dermatologie feiert Ihren 40. Geburtstag unter diesem Namen. Sie ist der Nachfolger der zweitältesten deutschen dermatologischen Zeitschrift, den Monatsheften für Praktische Dermatologie und zählt somit insge-

Wir schreiben das Jahr 2014 und es steht der 40. Geburtstag der Aktuellen Dermatologie an. Zu diesem Anlass erscheint wie bereits zum 30.Geburtstag ein Festband [1], dieses Mal ein Festheft. Die Zeitschrift Aktuelle Dermatologie wurde im Jahr 1974 gegründet und ist im Jahr 1975 erstmals erschienen ( Abb.1). Die Väter der Zeitschrift und somit die Gründungsherausgeber sind Herr Prof. Georg Brehm, Herr Prof. Hans-Joachim Heite und Herr Prof. Hansotto Zaun ( $\bullet$ Tab. 1; $\bullet$ Abb. 24). Die Schriftleitung übernahm bis 2001 Prof. G. Brehm.

Die Wurzeln reichen jedoch noch tiefer. Eigentlich ist zum 132-jährigen Bestehen zu gratulieren. Die Aktuelle Dermatologie wurde 1999 mit der Zeitschrift für Dermatologie vereinigt, deren Wurzeln wie Pfahlwurzeln sehr tief aber auch weit in die deutschsprachige Dermatologie reichen. Der Werdegang begann mit den Monatsheften für Praktische Dermatologie (gegründet 1882) unter der Redaktion von H. von Hebra (Wien), O. Lassar (Berlin), P.G. Unna (Hamburg). Der Verleger war Leopold Voss (Hamburg/Leipzig). Es ist die zweitälteste dermatologische Zeitschrift [2].

Zum Vergleich und für die zeitliche Einordnung: Filipp Josef Pick (Prag) und Heinrich Auspitz, (Wien) starteten 1869 das Journal Archiv für Dermatologie und Syphilis, die älteste Zeitschrift der deutschsprachigen Dermatologie [3], die Pick als Herausgeber bzw. Mitherausgeber bis zu seinem Tod betreute. Eine kurze Zeit wurde es Vierteljahresschrift genannt und dann unter dem „amerikanisierten“ Namen Archives of Dermatological Research weitergeführt [4]. Gemeinsam mit samt 132 Jahre, gegründet 1882 unter dem Namen Monatshefte für Praktische Dermatologie von H. von Hebra (Wien), O. Lasser (Berlin) und P.G. Unna (Hamburg). Sie befasst sich kontinuierlich und intensiv mit der relevanten wissenschaftlichen Literatur der Dermatologie und der benachbarten Fachgebiete.

Albert Neisser (Breslau) gründete Pick 1888 die Deutsche Dermatologische Gesellschaft, die dann 1889 registriert wurde [5]. Neisser initiiert 1902 im Roten Rathaus in Berlin die Deutsche Gesellschaft zur Bekämpfung der Geschlechtskrankheiten und eine Zeitschrift, die die Gesellschaft
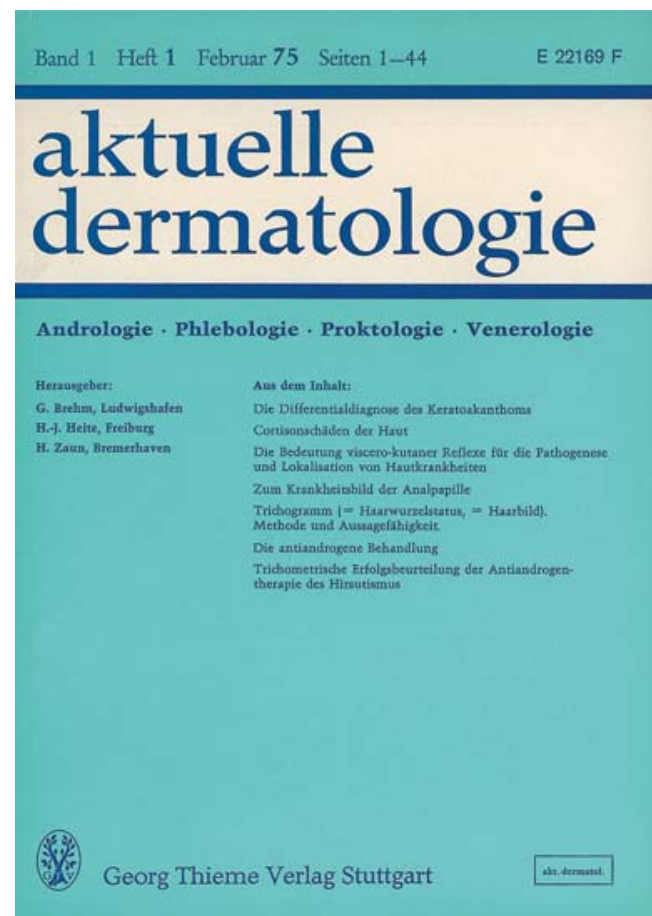

Abb.1 Die Umschlagseite des ersten Heftes von 1975 [1]. 
Tab. 1 Die Herausgeber der Aktuellen Dermatologie.

\begin{tabular}{|l|l|}
\hline Die Gründer 1975 & \\
\hline Georg Brehm, Ludwigshafen & $1975-2001$ \\
\hline Hans-Joachim Heite, Freiburg & $1975-1985$ \\
\hline Hansotto Zaun, Homburg & $1975-1999$ \\
\hline Später kamen dazu & \\
\hline Ernst G. Jung, Heidelberg, Mannheim & ab 1977 \\
\hline Manfred Goos, Essen & $1984-1991$ \\
\hline Peter Elsner, Jena & ab 1994 \\
\hline Eva-Bettina Bröcker, Würzburg & $1996-2000$ \\
\hline Rudolf Stadler, Minden & ab 1998 \\
\hline Hans Meffert, Berlin & ab 1999 \\
\hline Thomas Bieber, Bonn & $2001-2004$ \\
\hline Sergij Goerdt, Mannheim & $2002-2011$ \\
\hline Ingrid Moll, Hamburg & ab 2003 \\
\hline Peter von den Driesch, Stuttgart & ab 2004 \\
\hline Christiane Bayerl, Wiesbaden & ab 2006 \\
\hline Volker Mielke, Hamburg & ab 2006 \\
\hline Christos C. Zouboulis, Dessau & ab 2006 \\
\hline Kerstin Jung, Erfurt & ab 2010 \\
\hline Thomas Vogt, Homburg & ab 2014 \\
\hline Redaktion und Schriftführung lagen bei & \\
\hline Georg Brehm & $1975-2001$ \\
\hline Rudolf Stadler & $2002-2003$ \\
\hline Ernst G. Jung & $2004-2006$ \\
\hline Christiane Bayerl & ab 2007 \\
\hline
\end{tabular}

repräsentierte und später durch die Zeitschrift für die Bekämpfung der Geschlechtskrankheiten ersetzt wurde.

Die Monatshefte für Praktische Dermatologie gingen über in die Dermatologische Wochenschrift (1912) mit den Herausgebern P.G. Unna (Hamburg), J.H. Rille (Leipzig) und E. Delbanco (Hamburg). In den Jahren 1944-1948 wurde der Druck eingestellt. Die Wiederaufnahme der Ausgabe übernahm danach der Verleger Johann Ambrosius Barth (Leipzig) mit den Herausgebern L. Höhe (Berlin), C. Moncorps (Münster i.W.), J. Hämel (Jena), J.H. Rille (Innsbruck), H.A. Gottron (Tübingen) und W. Schönfeld (Heidelberg). Seit 1961 war E. Keining (Mainz) der alleinverantwortliche Redakteur. Im Jahre 1969 wurde die Zeitschrift zum offiziellen Organ der Gesellschaft für Dermatologie der Deutschen Demokratischen Republik ernannt und in Dermatologische Monatsschrift, mit dem Herausgebern W. Gertler (Berlin), H.E. Kleine-Natrop (Dresden) und N. Sönnichsen (Jena), umbenannt (৫ Abb.5). Ab 1976 war N. Sönnichsen (Berlin) allein verantwortlicher Chefredakteur. Nach einer weiteren Umbenennung zur Zeitschrift für Dermatologie im Jahre 1994 wurde sie 1999 mit der Aktuellen Dermatologie unter deren Namen zusammengefasst. Die Schriftleitung blieb bis 2001 bei Prof. G. Brehm, ihm folgten 2002-2003 Prof. R. Stadler (Minden), 2004-2006 Prof. E. G. Jung (Mannheim) und ab 2007 Frau Prof. C. Bayerl (Wiesbaden).

In der heutigen Aktuellen Dermatologie werden alle Artikel zusätzlich mit Titel und Abstract in Englisch geführt, die allen Internet-Nutzern frei zugänglich sind. Publiziert werden mittels PeerReview-Verfahren begutachtete Manuskripte zu originärer Forschung, Artikel für die kontinuierliche Medizinische Schulung (CME), ein Quiz zur Dermatohistopathologie, Übersichtsarbeiten sowie medizinhistorische Arbeiten und Kasuistiken. Zum Anreiz für junge Autoren wird jährlich ein Kasusitikpreis für die beste Arbeit des Jahres ausgeschrieben.

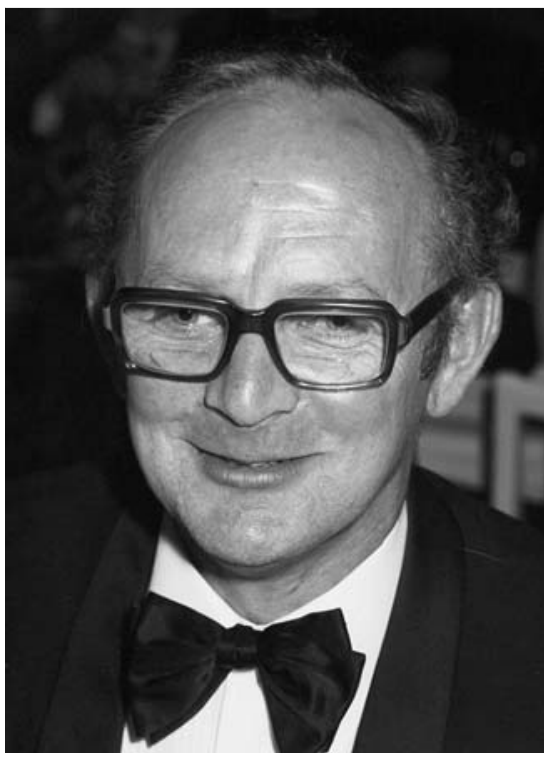

Abb.2 Georg Brehm, geb. 25. 10.1924, Gründungsherausgeber, Schriftleiter und Redaktion vom Anfang der Zeitschrift an, 27 Jahre lang bis 2001. Ludwigshafen a. Rh., Aufnahme 1976 [1].

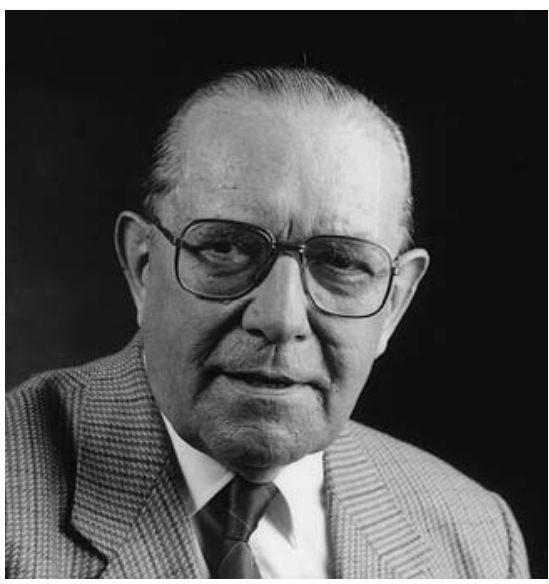

Abb.3 Hans-Joachim Heite, geb. 27. 8. 1913, gest. 28.11.1996.

Gründungsherausgeber bis 1985, Freiburg i. Br., Aufnahme 1993 [1].

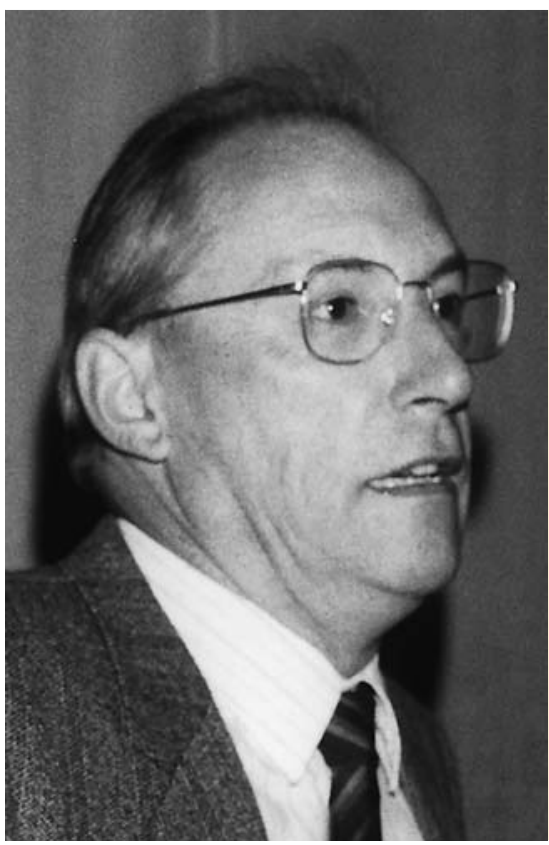

Abb.4 Hansotto Zaun, geb. 17. 10.1930, Gründungsherausgeber bis 1999. Homburg Saar, Aufnahme 1990 [1]. 


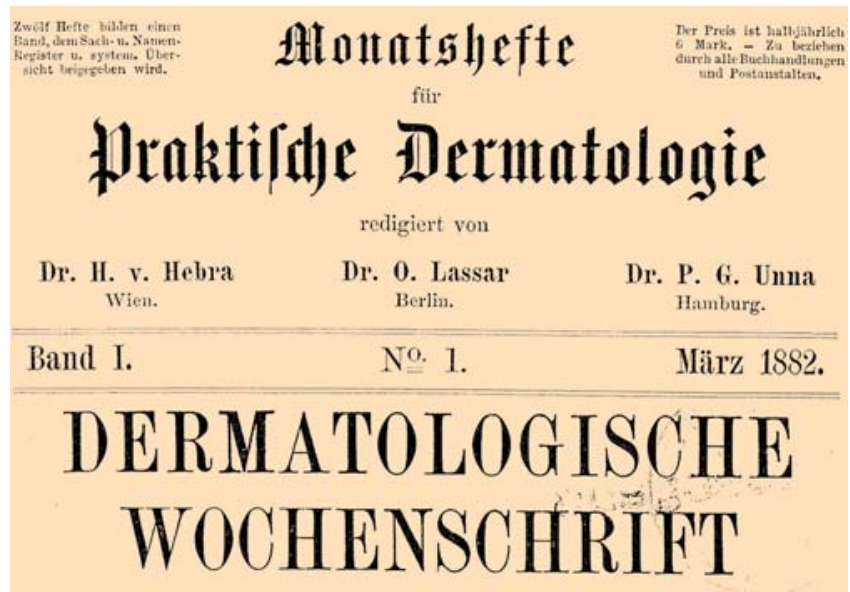

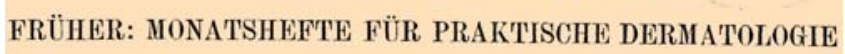
Dermatologifhe Wochenfhurift 20. Januar 1912 Nr. 3 Band 54

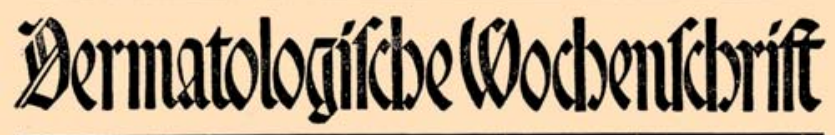

Bd. 100, Nr. 1

5. Januar 1935

\section{DERMATOLOGISCHE WOCHENSCHRIFT}

\section{Dermatologische Monatsschriit}

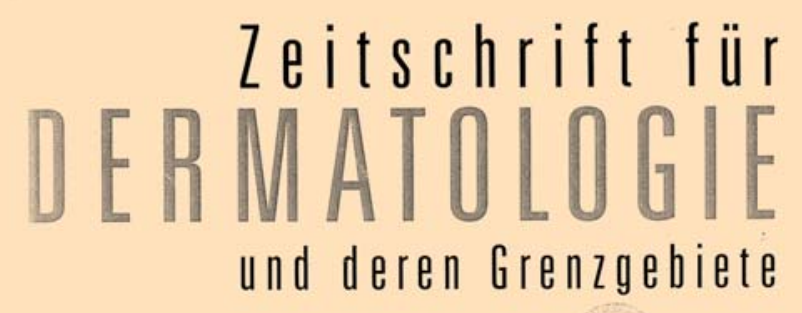

Abb.5 Titelzeilen der Zeitschrift für Dermatologie über die Jahre [1].
Im aktuellen Format umfasst die Zeitschrift folgende Rubriken:

- Spotlight

- Editorial

- Derma-Fokus

- Übersichten/Originalarbeiten

- Kasuistik (verantwortet von Herrn Prof. P. von den Driesch, Stuttgart)

- Von den Wurzeln unseres Fachs

- Fort- und Weiterbildung (CME) in jedem 2. Heft (in den Jahren 2007 - 2010 verantwortet von Herrn Prof. Zillikens, Lübeck, seit 2011 von Herrn Prof. E. Schultz, Nürnberg)

- Histologisches Quiz in jedem 2. Heft (verantwortet von Frau Prof. I. Moll, Hamburg, und Herrn Dr. Mielke, Hamburg)

- Buchbesprechungen

- Veranstaltungen

- Medizin \& Markt

Eine „Klinik im Blickpunkt“ gibt den Universitätskliniken und städtischen Häusern ein Forum, ihre Klinik mit Historie, aktueller Ausrichtung, Forschungsschwerpunkten, klinischer Arbeit und den für die Aktuelle Dermatologie so typischen Kasuistiken darzustellen.

Schwerpunkthefte zu aktuellen Themen ermöglichen es Gastherausgebern ihren Arbeitsbereich mit eingeladenen Manuskriptautoren komprimiert und zentriert darzustellen.

Der Verlag hat für die Leser als elektronischen Zusatznutzen geschaffen:

- Thieme Connect (seit 2001) mit PDF- und HTML-Versionen

- eFirst seit Juni 2007

- Online-CME-Portal seit 2008

Die Thieme Verlagsgruppe hat Standorte in Stuttgart, Berlin, Düsseldorf, Erlangen, New York, Delhi und seit neustem auch in Rio De Janeiro. Sie hat ihr 125-jähriges Jubiläum im Jahr 2011 (gegründet 1886 von Georg Thieme in Leipzig) gefeiert ( $\bullet$ Abb.6). Der Thieme Verlag gibt 4400 Buchtitel, Lehrbücher, Atlanten, Monografien, Nachschlagewerke und Ratgeber heraus und fast 150 Fachzeitschriften. Dr. Albrecht Hauff ist der Verleger der Thieme Verlagsgruppe und seit 1990 persönlich haftender Gesellschafter.

Die Zeitschrift ist im Ranking der Journale eine der meistgelesenen, auch unter den jungen Klinikärzten und niedergelassenen Kollegen - das ist der beste Lohn für die Herausgeber.

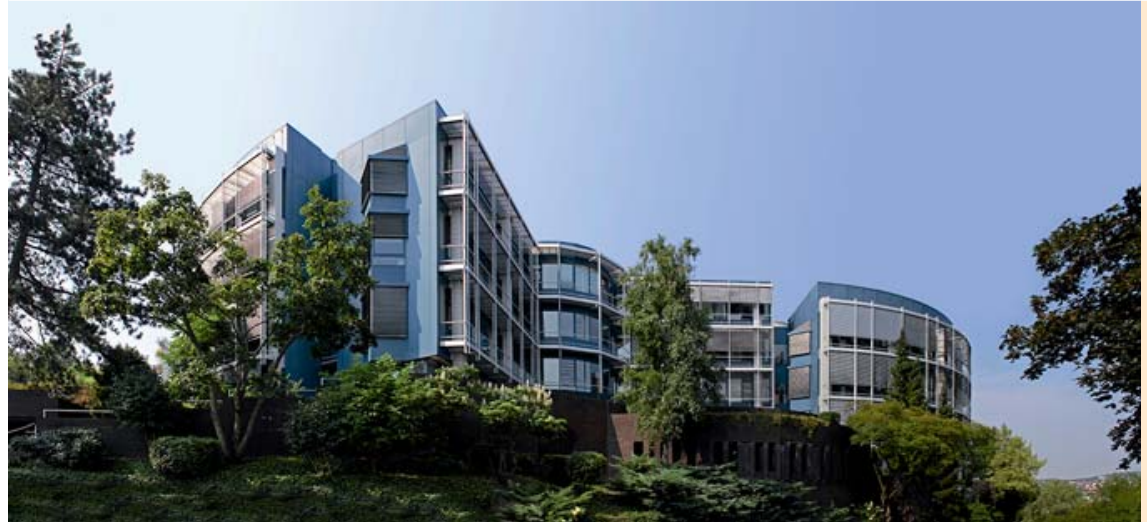

Abb. 6 Thieme Verlagsgebäude (Bild: Michael Zimmermann/Thieme). 


\section{Abstract}

\section{Years of Aktuelle Dermatologie}

$\nabla$

The Journal Aktuelle Dermatoloie celebrates the 40th anniversary, while originally dating back 132 years to Monatshefte für Praktische Dermatologie, founded 1882 by the editors H. von Hebra (Vienna), O. Lassar (Berlin) and P.G. Unna (Hamburg). It continuously and intensively deals with the relevant scientific and clinically informative literature of dermatology and the neighbouring fields.

\section{Literatur}

1 Jung EG, Hrsg. 30 Jahre Aktuelle Dermatologie, ein Jubiläumsband. Stuttgart: Thieme; 2005

2 Scholz A. Oscar Lassar. In: Löser C, Plewig G, Burgdorf WHC, Hrsg. Pantheon of Dermatology. Heidelberg: Springer; 2013

3 Arenberger P, Kulichová D. Filipp Josef Pick. In: Löser C, Plewig G, Burgdorf WHC, Hrsg. Pantheon of Dermatology. Heidelberg: Springer; 2013: 858

4 Hundeiker M. Heinrich Auspitz. In: Löser C, Plewig G, Burgdorf WHC, Hrsg. Pantheon of Dermatology. Heidelberg: Springer; 2013: 51

5 Scholz A. Albert Neisser. In: Löser C, Plewig G, Burgdorf WHC, Hrsg. Pantheon of Dermatology. Heidelberg: Springer; 2013: 774 